\title{
30100 A Case of Wide Necked Basilar Tip Technical Aneurysm Treated with Horizontal Stent-assisted Coil Embolization
}

Kosuke Kumagai, ${ }^{1}$ Kouhei Nii, ${ }^{2}$ Hayatsura Hanada, ${ }^{2}$ Ayumu Eto, ${ }^{2}$ Takafumi Mitsutake, ${ }^{2}$ Ritsurou Inoue, ${ }^{2}$ Kimiya Sakamoto, ${ }^{2}$ Masanori Tsutsumi, ${ }^{2}$ Minoru Iko, ${ }^{2}$ Hiroshi Aikawa, ${ }^{2}$ and Kiyoshi Kazekawa ${ }^{2}$

Objectives: We report our experience with a patient undergoing horizontal stent-assisted coil embolization for an aneurysm of the basilar tip, in which favorable coil embolization was achieved through innovative approaches that enhanced our intraoperative working views.

Case Presentation: A 50-year-old woman was referred to our institution for further evaluation and treatment of an unruptured basilar tip aneurysm which had been diagnosed by magnetic resonance angiography (MRA). A cerebral angiogram showed a wide-necked saccular aneurysm (7.2-mm diameter/6.8-mm neck length), and bilateral posterior cerebral arteries (PCAs) arising directly from the aneurysmal neck. A right internal carotid artery (ICA) angiograms revealed a relatively developed right posterior communicating artery (PcomA) (1.1-mm diameter), and there was neither severe vascular bending nor tortuosity in the areas encompassing the right PcomA and the left P1 and P2 of PCA. We performed horizontal stent-assisted coil embolization via the right PcomA, because horizontal stent-assisted coil embolization was possible to perform anatomically and was considered safer and simpler than other adjunctive techniques. Simultaneous internal carotid-vertebral angiograms allowed stent delivery systems to be navigated from the right ICA to the left PCA via the right PcomA using fewer steps.

Conclusions: If the anatomy would be favorable, horizontal stent-assisted coil embolization could be considered as an alternative method, in the case of other adjunctive techniques being difficult to perform for a wide-necked basilar tip aneurysm. Innovative approaches designed to enhance an appropriate intraoperative working view would facilitate performing horizontal stent-assisted coil embolization.

Keywords > horizontal, basilar tip aneurysm, barrel view, stent-assisted coil embolization, simultaneous internal carotidvertebral angiogram

\section{Introduction}

For wide-necked aneurysms, endovascular treatment combined with adjunctive techniques using balloons or stents have achieved good outcomes. ${ }^{1-3)}$ In Japan as well, since the introduction of self-expandable stents designed to augment endovascular treatment, stent-assisted coil embolization

${ }^{1}$ Department of Neurosurgery, National Defense Medical College Hospital, Tokorozawa, Saitama, Japan

${ }^{2}$ Department of Neurosurgery, Fukuoka University Chikushi Hospital, Chikushino, Fukuoka, Japan

Received: November 12, 2015; Accepted: February 10, 2016 Corresponding author: Kosuke Kumagai. Department of Neurosurgery, National Defense Medical College Hospital, 3-2 Namiki, Tokorozawa-shi, Saitama, 359-8513, Japan

Email: kumagaikousuke0714@yahoo.co.jp

(C)2016 The Editorial Committee of Journal of Neuroendovascular Therapy. All rights reserved. has tended to be selected. However, some wide-necked aneurysms have a shape that makes it difficult to insert a coil into the aneurysm when employing stent-assisted coil embolization with conventional stent placement. Thus, various innovative approaches for stent placement, such as multiple stent placements, the waffle-cone stent technique, and the horizontal stent technique, have been attempted in patients with such aneurysms. ${ }^{4-12)}$

We herein report our experience with a patient undergoing horizontal stent-assisted coil embolization for an aneurysm of the basilar tip, in which favorable coil embolization was achieved through innovative approaches that enhanced an appropriate intraoperative working views.

\section{Case Presentation}

Patient: A 50-year-old woman

Past history: Hypertension 


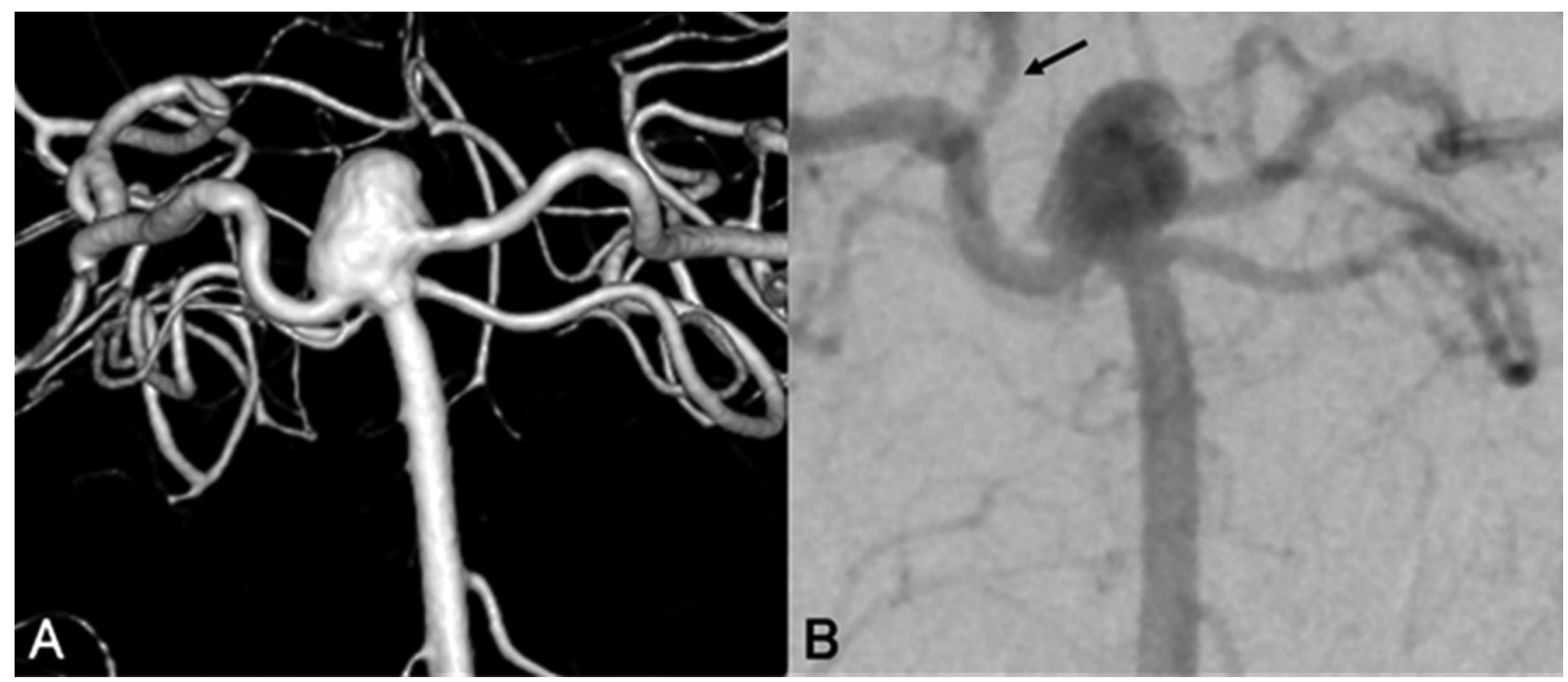

Fig. 1 (A) A three-dimensional rotational view of the preoperative right vertebral angiogram shows a basilar tip aneurysm with a maximum diameter of $6.8 \mathrm{~mm}$ and a neck diameter of $7.2 \mathrm{~mm}$. The bilateral posterior cerebral arteries arise directly from the aneurysmal neck and they arise from the basilar artery at a nearly right angle. (B) The antero-posterior view of the Allcock test (the right vertebral angiogram was obtained with right carotid artery compression) demonstrates the relatively developed right posterior communicating artery (arrow).

History of present illness: She was referred to our institution for further evaluation and treatment of an unruptured basilar tip aneurysm which had been diagnosed by magnetic resonance angiography (MRA).

Imaging findings on admission: Preoperative angiography revealed a saccular aneurysm with a maximum diameter of $6.8 \mathrm{~mm}$ and a neck diameter of $7.2 \mathrm{~mm}$ at the tip of the basilar artery (BA). Bilateral posterior cerebral arteries (PCAs) were observed to arise directly from the aneurysm (Fig. 1). An internal carotid angiography (ICAG) revealed a well developed right posterior communicating artery (PcomA) with a diameter of $1.1 \mathrm{~mm}$.

Endovascular procedure: According to the patient's request, endovascular treatment for the basilar tip aneurysm was scheduled. For preparing to stent-assisted coil embolization, antiplatelet therapy (aspirin, $100 \mathrm{mg} /$ day) was started 2 weeks before the procedure. In consideration of the complicated configuration of the aneurysm, we decided to treat the aneurysm with horizontal stent-assisted coil embolization through the right PcomA.

A 6-Fr sheath was inserted into the right femoral artery, and a 5-Fr sheath was inserted into the left femoral artery. A 6-Fr guiding catheter (Roadmaster TH; Goodman, Aichi, Japan) was placed in the right vertebral artery (VA) via the right femoral artery. Then, a 5-Fr guiding catheter (Guider Softip; Stryker, Kalamazoo, MI, USA) was placed in the right internal carotid artery (ICA) via the left femoral artery. Echelon-10 (eV3 Neurovascular, Irvine, CA, USA) was placed in the BA, road-map images were constructed from the antero-posterior view and the lateral view while nonionic iodinated contrast medium was simultaneously injected from the ICA and the VA. Using these road-map images as a guide, we advanced a Prowler Select Plus (Cordis, Johnson \& Johnson, Fremont, CA, USA) over a 200-cm-in-length CHIKAI black (shaft diameter: 0.014 inch, tip shape: $90^{\circ}$; Asahi Intec, Aichi, Japan) from the right ICA through the right PcomA and the right P1 of PCA to the left PCA (Figs. 2A-2D). Echelon-10 was carefully advanced into the aneurysm, and then approximately two loops of Axium 3D $6 \mathrm{~mm} \times 15 \mathrm{~cm}$ (eV3 Covidien, Irvine, CA, USA) were then advanced within the aneurysm. Enterprise vascular reconstruction device (VRD) of the non-distal tip type, $4.5 \mathrm{~mm}$ in diameter $\times 22 \mathrm{~mm}$ in length (Johnson \& Johnson, Miami, FL, USA), was deployed from the proximal segment of the left P2 segment to just proximal to the right $\mathrm{P} 1 / \mathrm{P} 2$ junction with sufficient coverage of the aneurysmal neck (Fig. 2E). Under the downthe-barrel view constructed with lateral fluoroscopic image, further coil embolization could be performed through the jailing approach without coil migration or neck remnant (Figs. 3A-3C).

Postoperative course: Systemic heparinization was started after surgery. On the following day, clopidogrel ( $50 \mathrm{mg} /$ day) and cilostazol (200 mg/day) were added to initiate 3-drug antiplatelet therapy. Follow-up angiogram 3 months after the procedure revealed complete occlusion of the aneurysm and patency of all the branches (Fig. 3D). The patient was 


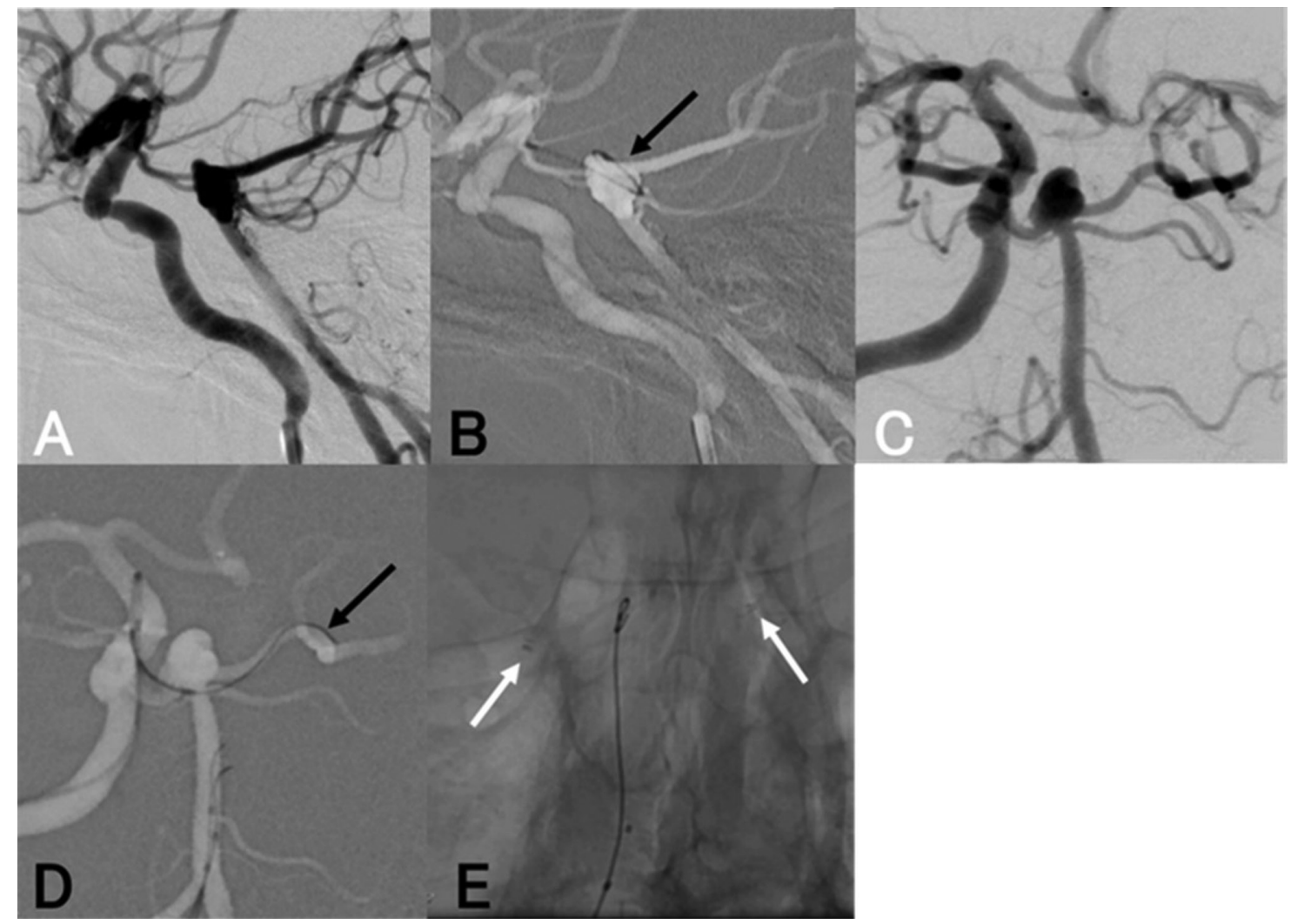

Fig. 2 The lateral view of the simultaneous internal carotid-vertebral angiography (IC-VAG) (A) and road mapping image (B) shows the course of the micro-catheter from the right internal carotid artery, through the right posterior communicating artery (PcomA), and the right posterior cerebral artery (PCA) (black arrow). The antero-posterior view of the simultaneous IC-VAG (C) and road mapping image (D) show the course of the micro-catheter from the right PCA to the left P2 of PCA (black arrow). (E) Enterprise VRD (no distal type) was deployed across the aneurysmal neck with both markers (white arrows) in both PCAs. VRD: vascular reconstruction device

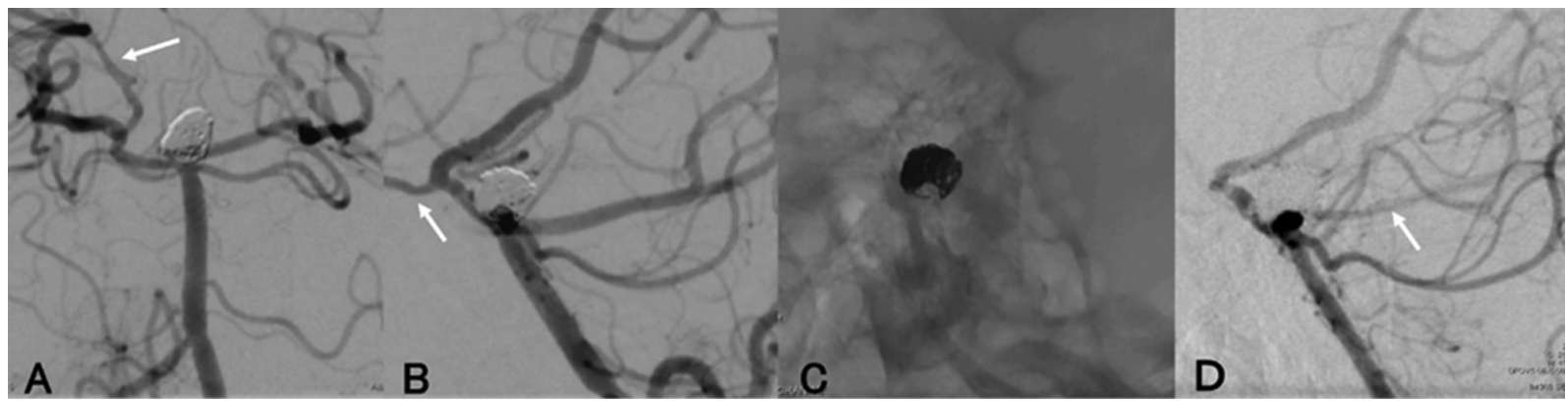

Fig. 3 The post-coiling angiogram (A) is an antero-posterior view of the right vertebral angiogram showing the patency of both posterior cerebral arteries (PCAs) and the right posterior communicating artery (PcomA) (arrow). There was no coil protrusion. (B) The down-the-barrel view of the right vertebral angiogram shows neither coil protrusion nor any neck remnants; also, both PCAs and the right PcomA (arrow) are confirmed to be patent. (C) The down-the-barrel view shows the patency of the stent lumen and complete occlusion of the aneurysm. (D) Follow-up angiograms at 3 months show the patency of both PCAs and the stent lumen.

doing well and the antiplatelet regimen has been reduced to monotherapy with aspirin.

\section{Discussion}

Various innovative approaches for stent-assisted coil embolization have been reported, including the Y-configuration, waffle-cone, and horizontal stent techniques. The Yconfiguration stent technique includes kissing, ${ }^{4)}$ crossing, ${ }^{4-6)}$ and non-overlapping approaches. ${ }^{7}$ As all of these techniques involve multiple stent placements, there are some drawbacks, such as the increasing probability of thrombogenicity and high costs., ${ }^{3,5)}$ Waffle-cone stent technique consists the distal end of the stent positioned adjacent 
to the aneurysmal neck to prevent coil migration to the parent artery. Although this technique is considered to be useful for supporting the coil mass with one stent, ${ }^{8,9}$ ) it is considered to be unsuitable for patients whose aneurysms have a narrow neck (less than $4.5 \mathrm{~mm}$ ) or a low dome height. ${ }^{8)}$

The horizontal stent technique is a method for horizontally placing a stent across the aneurysmal neck of a bifurcation type aneurysm through the anterior communicating artery or PcomA. ${ }^{10-12)} \mathrm{A}$ problem with this technique is that the approaches to the lesion are restricted by the anatomical characters. ${ }^{11,12)}$ Several factors are considered to be important when horizontal stent-assisted coil embolization is performed for a basilar tip aneurysm, as follows; the absence of severe bending or tortuosity of the PcomA or bilateral PCAs, which serve as the access routes, the angle formed by the BA and the bilateral P1, and the diameter of the PcomA (1 mm or more). Previous reports also stressed failure of horizontal stent-assisted coil embolization in some cases, because of an inability to guide a micro-catheter to the contralateral PCA due to access route problems. $\left.{ }^{11}\right)$

In our case, as the bilateral PCAs arose directly from the cerebral aneurysm, there would be a risk of coil migration to the parent artery if the simple technique, balloon neck plasty, or the double catheter technique had been applied. We considered horizontal stent-assisted coil embolization feasible, because of the anatomical characters of the lesion, such as the well-developed right PcomA with a diameter of $1.1 \mathrm{~mm}$, and absence of severe vascular bending or tortuosity of arteries involved. Horizontal stent-assisted coil embolization was also considered simpler and safer than other adjunctive techniques in consideration of accessibility to the lesion and thrombogenicity.

In order to perform successful horizontal stent-assisted coil embolization in this patient, optimal intraoperative working views and selecting the appropriate devices were mandatory.

A road-map image was constructed by simultaneous angiography of the ICA and VA under biplane fluoroscopic guidance. In horizontal stent-assisted coil embolization for the basilar tip aneurysm, it was necessary to understand the anatomical relationships including the course of the PcomA in the lateral view of the ICAG and the locations of the bilateral PCAs and the aneurysmal neck in the antero-posterior view of the vertebral angiograms (VAGs). A pair of roadmap images (an antero-posterior and a lateral view) constructed by simultaneous carotid-vertebral angiography (IC-VAG) allowed navigation of the micro-guidewire and catheter to the appropriate position for the deployment of a horizontal stent, and delivering coils. Simultaneous IC-VAG helped reducing the frequency of angiographies and facilitating the procedure.

Enterprise VRDs include the non-distal tip type device that has a short tip on the delivery wire. It allows a stent to be placed from a more proximal part of the parent artery without guiding a micro-catheter to a distal segment of the PCA, and it also reduces the risk of vascular injury. In the present case, the use of the non-distal tip type device allowed us to place the stent in the appropriate position, from the proximal segment of the left $\mathrm{P} 2$ segment to just proximal to the right $\mathrm{P} 1 /$ P2 junction. As for catheterization to the dome of the aneurysm, the trans-cell approach for horizontal stent-assisted coil embolization requires the catheter passing through stent struts twice before reaching the dome of the aneurysm. In order to overcome these disadvantages, we used the jailing technique, which was considered simpler and safer than the trans-cell approach. With the jailing technique, uneventful catheterization and coil deliveries could be achieved. The lateral fluoroscopy image as the down-the-barrel view provided excellent visualization of the parent arteries and the aneurysm with preventing coil migration or remnant of the neck. ${ }^{13)}$

Regarding the long term results of horizontal stent-assisted coil embolization, no consensus has been yet reached because of limited number of cases reported. Although it has been indicated that horizontal stent-assisted coil embolization is less effective for reducing wall shear stress in an aneurysm than the multiple stent placement technique, ${ }^{10)}$ tight coil packing may prevent aneurysmal recanalization.

\section{Conclusion}

We experienced a case in which horizontal stent-assisted coil embolization was performed for a wide necked basilar tip aneurysm. This technique appeared to be effective and safe for a wide-necked basilar tip aneurysm from which the bilateral PCAs arised directly. It is necessary to sufficiently assess the access route and the anatomical relationship between involving arteries and the aneurysm when planning the procedure.

\section{Disclosure Statement}

The authors have no personal, financial, or institutional interest in any of the drugs, materials, or devises used in this article. All authors who are members of the Japan Neurovascular Society have registered online self-reported COI Disclosure Statement Forms. 


\section{References}

1) Moret J, Cognard C, Weill A, et al: Reconstruction technic in the treatment of wide-neck intracranial aneurysms. Longterm angiographic and clinical results. Apropos of 56 cases. J Neuroradiol 1997; 24: 30-44.

2) Chalouhi N, Jabbour P, Singhal S, et al: Stent-assisted coiling of intracranial aneurysms: predictors of complications, recanalization, and outcome in 508 cases. Stroke 2013; 44: 1348-1353.

3) Fiorella D, Albuquerque FC, Deshmukh VR, et al: Usefulness of the Neuroform stent for the treatment of cerebral aneurysms: results at initial (3-6-mo) follow-up. Neurosurgery 2005; 56: 1191-1201; discussion 1201-1202.

4) Zhao KJ, Yang PF, Huang QH, et al: Y-configuration stent placement (crossing and kissing) for endovascular treatment of wide-neck cerebral aneurysms located at 4 different bifurcation sites. AJNR Am J Neuroradiol 2012; 33: 1310-1316.

5) Thorell WE, Chow MM, Woo HH, et al: Y-configured dual intracranial stent-assisted coil embolization for the treatment of wide-necked basilar tip aneurysms. Neurosurgery 2005; 56: 1035-10340; discussion 1035-1040.

6) Chow MM, Woo HH, Masaryk TJ, et al: A novel endovascular treatment of a wide-necked basilar apex aneurysm by using a Y-configuration, double-stent technique. AJNR Am J Neuroradiol 2004; 25: 509-512.

7) Cho YD, Park SW, Lee JY, et al: Nonoverlapping Yconfiguration stenting technique with dual closed-cell stents in wide-neck basilar tip aneurysms. Neurosurgery 2012; 70: 244-249.

8) Padalino DJ, Singla A, Jacobsen W, et al: Enterprise stent for waffle-cone stent-assisted coil embolization of large widenecked arterial bifurcation aneurysms. Surg Neurol Int 2013; 4: 9 .

9) Horowitz M, Levy E, Sauvageau E, et al: Intra/extraaneurysmal stent placement for management of complex and wide-necked-bifurcation aneurysms: eight cases using the waffle cone technique. Neurosurgery 2006; 58(4 Suppl 2): ONS-258-ONS-262.

10) Kono $K$, Terada $T$. Hemodynamics of 8 different configurations of stenting for bifurcation aneurysms. AJNR Am J Neuroradiol 2013; 34: 1980-1986.

11) Blackburn SL, Kadkhodayan Y, Shekhtman E, et al: Treatment of basilar tip aneurysms with horizontal PCA to PCA stent-assisted coiling: case series. J Neurointerv Surg 2013; 5: 212-216.

12) Siddiqui MA, J Bhattacharya J, Lindsay KW, et al: Horizontal stent-assisted coil embolisation of wide-necked intracranial aneurysms with the Enterprise stent-a case series with early angiographic follow-up. Neuroradiology 2009; 51: 411-418.

13) Fiorella D, Albuquerque FC, Masaryk TJ, et al: Balloon-instent technique for the constructive endovascular treatment of "ultra-wide necked" circumferential aneurysms. Neurosurgery 2005; 57: 1218-1227; discussion 1218-1227. 